\title{
Acute Toxicity of Three Dimensional Conformal Concurrent Chemoradiation with Dose Optimization for External Beam Radiation in Carcinoma Cervix-A Prospective Study
}

\author{
Sathish Balachandran1, Shyama Prem Sudha ${ }^{1 *}$, Reddy KS ${ }^{2}$, Saravanan Kandasamy ${ }^{3}$ \\ ${ }^{1}$ Department of Radiotherapy, JIPMER, Puducherry, India \\ ${ }^{2}$ Department of Radiotherapy, MGMC, Puducherry, India \\ ${ }^{3}$ Department of Medical Physics, JIPMER, Puducherry, India \\ Email: sathishbalachandran@gmail.com, "shyamagopalakrishnan@yahoo.co.in, atsahara11@yahoo.com, \\ kandasamysaravanan@yahoo.com
}

Received 29 June 2016; accepted 28 August 2016; published 31 August 2016

Copyright (C) 2016 by authors and Scientific Research Publishing Inc.

This work is licensed under the Creative Commons Attribution International License (CC BY). http://creativecommons.org/licenses/by/4.0/

(c) (†) Open Access

\section{Abstract}

Aim: To prospectively evaluate the acute toxicity of 3D conformal radiotherapy with dose optimization in patients with carcinoma cervix. Materials and Methods: Carcinoma cervix patients stage IIB to IIIB (n = 30) treated during November 2011 to May 2013 at the institution with 3D conformal chemo-radiation were included in the study. They received weekly Cisplatin $40 \mathrm{mg} / \mathrm{m}^{2}$ for a maximum of 5 cycles. They received $46 \mathrm{~Gy} / 23$ fractions, 5 fractions per week of external beam radiation. In these patients dose optimization was done in order to achieve a tumor maximum dose $\left(D_{\max }\right)$ around $\mathbf{1 0 5 \%}$. Various techniques were used for dose optimization which included the use of sub fields, adjusting the weightages, using wedges and the use of mixed energies. EBRT (External Beam Radiotherapy) was followed by two fractions of high dose rate intracavitary brachytherapy of $9 \mathrm{~Gy}$ each. Acute RTOG toxicity was assessed weekly during EBRT and 1 week post EBRT. Results: The median age of the patients was 45 (range: 30 - 55 years). All the patients completed EBRT; 63.3\% of the patients received all 5 cycles of chemotherapy while $26.6 \%$ of the patients received 4 cycles of chemotherapy and $10 \%$ of the patients received 3 cycles of chemotherapy. The most predominant toxicity seen was GI toxicity, diarrhea being the most common GI toxicity followed by vomiting. Neutropenia was the most common hematological toxicity. Most patients had grade 0 and grade 1 toxicity. None of the patients had grade 4 toxicity while few had grade 2 and 3 toxicity. Conclusion:

\footnotetext{
"Corresponding author.
}

How to cite this paper: Balachandran, S., Sudha, S.P., Reddy KS and Kandasamy, S. (2016) Acute Toxicity of Three Dimensional Conformal Concurrent Chemoradiation with Dose Optimization for External Beam Radiation in Carcinoma Cervix-A Prospective Study. International Journal of Medical Physics, Clinical Engineering and Radiation Oncology, 5, $204-211$. http://dx.doi.org/10.4236/ijmpcero.2016.53022 
3D conformal concurrent chemo radiotherapy with $D_{\max }$ around $105 \%$ reduces acute $R T O G$ toxicity particularly grade 3 and 4 and improves patient compliance for concurrent chemo-radiotherapy.

\title{
Keywords
}

\author{
3D Conformal, Dose Optimization, Acute Radiation Toxicity
}

\section{Introduction}

Cervical cancer is the second most common cancer of women worldwide, with 471,000 incident cases estimated in 2000 and a 5-year prevalence of more than 1.4 million cases [1].

The two major modalities of treatment for carcinoma cervix are surgery and radiotherapy (with or without chemotherapy). Radiotherapy consists of External Beam Radiotherapy (EBRT) and brachytherapy.

Randomized controlled studies have reported a significant decrease in the pelvic nodal recurrence and distant metastasis in cancer cervix patients treated with chemoradiation [2]. Three-dimensional radiotherapy planning techniques, including conformal radiotherapy and intensity-modulated radiotherapy, have the potential for improving outcomes and reducing toxicities in cervical cancer.

Dosimetric comparisons have been done between conventional and 3DCRT/IMRT and have demonstrated a decrease in the radiation dose delivery to bowel, bladder and bone marrow and hence a decreased toxicity to these organs [3]. Forrest et al. found that the conventional 4 field box covered both the macroscopic and microscopic extent of the disease but because the treated volume is large the coverage of the PTV is achieved with considerable irradiation of normal tissues with significant acute as well as late toxicity particularly of the small bowel, sigmoid, rectum and bladder [4]. Gerstner et al. showed that there was a $10 \%$ reduction in small bowel volume which is inside the treated volume and a $20 \%$ reduction in the risk of geographical miss when dose distributions of conformal RT were compared with four field box techniques [5]. Published studies on acute toxicity and treatment tolerance of concurrent chemo radiation with 3D planning are largely lacking in literature. The objective of the 3DCRT planning in our study was to optimize the dose distribution in such a way that the PTV maximum dose is around $105 \%$ at the same time achieving 99\% coverage of PTV with the 99\% isodose line. We wanted to study if this dose optimization resulted in an improved acute toxicity profile. Since acute toxicity reflects tolerance and compliance of the patient to the treatment, measures to reduce acute toxicity will also result in an increase in the number of patients who complete their full treatment of radiation.

Hence our study aimed at evaluating the acute toxicity profile, feasibility and tolerance of chemo radiation in cervical cancer patients planned with three dimensional conformal radiotherapy with dose optimization.

\section{Materials and Methods}

\subsection{Patients}

We enrolled women of all ages who had stages IIB - IIIB squamous-cell carcinoma, of the cervix according to the staging system of the International Federation of Gynecology and Obstetrics 2009, with a ECOG score of 0 2 and normal blood counts, normal blood urea nitrogen, creatinine and bilirubin. Women were excluded from the study if they had IVA and IVB disease, Lower 1/3rd vagina involved by tumor, age more than 55, associated significant medical co morbidities, ovarian mass, preexisting gross pelvic inflammatory disease/tuberculosis of the genital tract, endometriosis and history of hemorrhoids/ulcerative colitis. Exclusion criteria also included any medical contraindications to chemotherapy and prior pelvic radiotherapy or systemic chemotherapy.

\subsection{Technique of EBRT}

All patients underwent CT simulation in the supine position with a comfortably full bladder. CT-simulation was performed using Siemens dual slice Somatom Spirit CT. CT scan of the abdomen and pelvis was performed from the level of L1 vertebral body to mid femur using $5 \mathrm{~mm}$ slice thickness with $3 \mathrm{~mm}$ reconstruction. The planning target volume (PTV) was contoured according to the radiation therapy planning consensus definitions of the Radiation Therapy Oncology Group RTOG)

[https://www.rtog.org/CoreLab/ContouringAtlases/GYN.aspx]. [6] Computerized treatment planning was done 
using Eclipse (Varian, USA) planning system. The Treatment Planning System (TPS) used AAA algorithm for calculation of 3DCRT plans. Two anterior and posterior fields and 2 lateral fields were used for treating the whole pelvis. Multi leaf collimators (120 leaf) were used for beam shaping and shielding with a beam energy of $6 \mathrm{MV} / 15 \mathrm{MV}$.

\subsection{Dose Optimization}

ICRU 62 accepts the dose variation to be within $\pm 5 \%$ to $7 \%$ [7]. Normally in 3 dimensional planning for cervix a four field box is designed and the isodose which covers the target volume adequately is chosen for normalization. However the absolute dose will then increase and the recalculated Dmax will be normally greater than $107 \%$. The objective of dose optimization for 3DCRT planning in our study was to optimize the dose distribution in such a way that the PTV maximum dose is around $105 \%$ while achieving $99 \%$ coverage of PTV with the $99 \%$ isodose line. This was achieved by first altering the weight age of beams. Then any areas of over dosage and under dosage was taken care of by inserting subfields i.e., field in field, adjusting the MLCs i.e., closing the high dose region with MLC. Further priming was done by inserting wedges and altering wedge angles.

After normalization if the initial Dmax of PTV if less than 105\% no further optimization was employed (Figure 1 and Figure 2). If it was more than 105\% the above mentioned methods were tried in an iterative fashion to reduce the Dmax. EBRT dose delivered was 46 Gy in 23 fractions, 1 fraction per day, and 5 fraction a week over 4 5 weeks. High dose rate brachytherapy using Iridium 192 was used to deliver two sessions of 9 Gy to Point A. The acute RTOG toxicity was analyzed weekly during EBRT and one week after completion of brachytherapy.

\subsection{Concurrent Chemotherapy}

All patients received weekly Cisplatin $40 \mathrm{mg} / \mathrm{m}^{2}$ for a maximum of 5 cycles.

\section{Statistical Tests}

Variables were expressed as mean and standard deviation. Chi square test was used for analysis of parameters. A p value of $<0.05$ considered significant. SPSS software was utilized to complete the statistical analysis. The study was cleared by the Institute ethics board and consent in vernacular language was obtained from all participants after proper explanation of the treatment options.

\section{Results}

30 consecutive patients with carcinoma cervix belonging to stage IIB to IIIB treated during November 2011 to

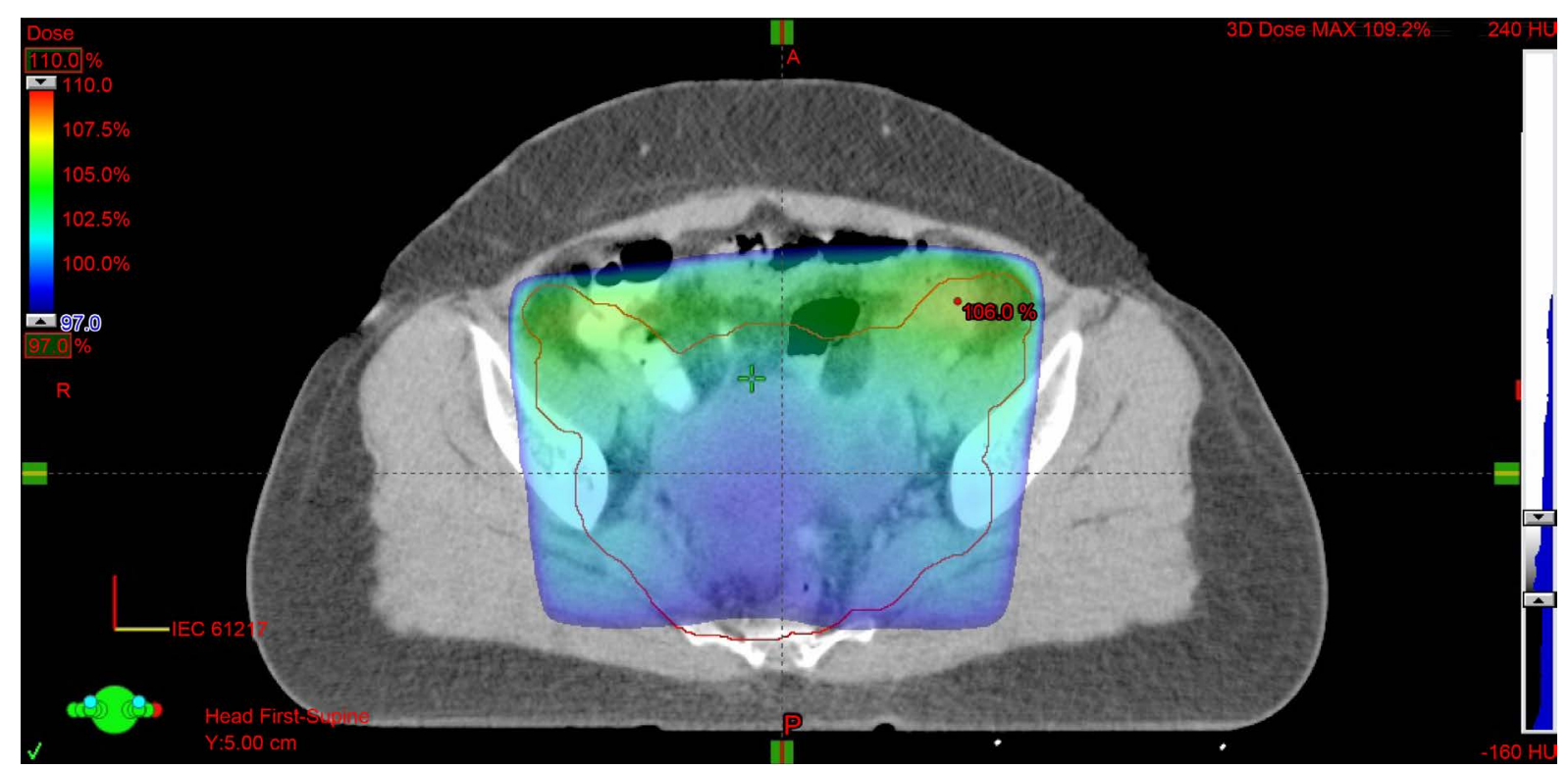

Figure 1. Pre-optimisation $\mathrm{D}_{\max } 109.2 \%$. 


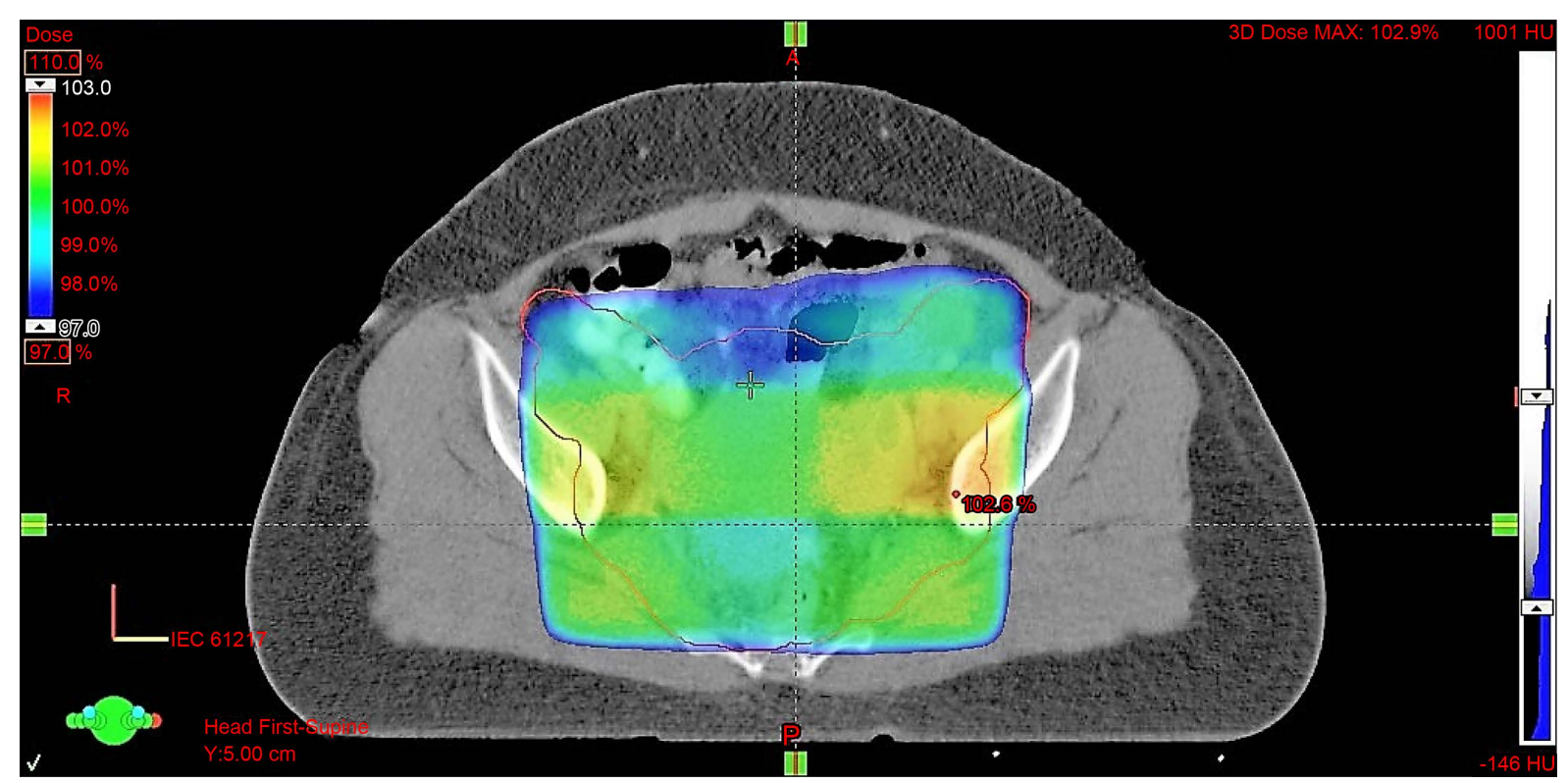

Figure 2. Post-optimisation $\mathrm{D}_{\max } 102.9 \%$.

May 2013 at the institution with 3D conformal chemo-radiation were included in the study. The median age of the patients was 45 (range 30 - 55). 80\% of patients had a pretreatment ECOG performance status (PS) $1 \%$ and $20 \%$ belonged to PS 2. Majority of the patients (66.67\%) had stage IIB and $33.33 \%$ had stage IIIB disease at presentation. $63.3 \%$ patients received the full 5 cycles of cisplatin while $26.6 \%$ and $10 \%$ received 4 and 3 cycles of chemotherapy respectively.

A total of 5 patients (16.67\%) had a delay in treatment. 3 patients had delay due to technical problems in (the LINAC machine and the delay was for less than 7 days. One patient developed grade 3 diarrhea (delay 10 days) and one patient developed grade 3 anemia (delay 9 days) due to which treatment was delayed for more than 1 week. The patients were given supportive care and radiation was continued with gap correction.

\subsection{Toxicity}

While $43.3 \%$ of patients had skin reactions $13.3 \%$ had vaginal mucosal toxicity and $3.3 \%$ had genitourinary toxicity.

GI toxicity appeared to the most predominant toxicity. The most frequently observed GI toxicity was diarrhea. $56.7 \%$ of patients developed diarrhea and $40 \%$ developed vomiting and $40 \%$ of patients developed rectal toxicity of any grade.

Overall $20 \%$ of the patients developed hematological toxicity of some grade. While neutropenia (20\%) was the most common hematological toxicity seen, only 1 patient developed anemia and 2 patients developed thrombocytopenia.

\subsection{Grade of Toxicity}

\section{1) Skin (Table 1)}

Out of 13 patients who developed skin reactions, 11 (84.62\%) had grade 1 and 2 (15.38\%) had grade 2 toxicity. None had grade 3 or 4 toxicities. The most common toxicity observed in the skin was hyperpigmentation.

2) Vaginal mucosa (Table 1)

All the four patients who developed vaginal mucosal toxicity had only grade 1 reaction in the form of vaginal injection/erythema. Vaginal toxicity developed after 2 weeks i.e., after $20 \mathrm{~Gy}$.

3) Gastrointestinal toxicity (Table 2)

Diarrhea:

Diarrhea was the most common toxicity observed. Of the patients who had diarrhea $64.71 \%$ had grade 1 , $23.53 \%$ had grade 2 requiring oral fluid and para sympatholytic drugs to control diarrhea and $11.76 \%$ had grade 
Table 1. Status of skin, vaginal and genitourinary toxicity.

\begin{tabular}{cccc}
\hline Parameter & Status of toxicity & Frequency & Percentage \\
\hline Skin & Grade 0 & 17 & 56.7 \\
& Grade 1 and above & 13 & 43.3 \\
& Total & 30 & 100 \\
Vaginal mucosa & Grade 0 & 26 & 86.7 \\
& Grade 1 and above & 4 & 13.3 \\
Genitourinary & Total & 30 & 100 \\
& Grade 0 & 29 & 96.7 \\
\hline
\end{tabular}

Table 2. Status of GI toxicity.

\begin{tabular}{|c|c|c|c|}
\hline Parameter & Status of toxicity & Frequency & Percentage \\
\hline \multirow[t]{3}{*}{ Diarrhea } & Grade 0 & 13 & 43.3 \\
\hline & Grade 1 and above & 17 & 56.7 \\
\hline & Total & 30 & 100 \\
\hline \multirow[t]{3}{*}{ Vomiting } & Grade 0 & 18 & 60 \\
\hline & Grade 1 and above & 12 & 40 \\
\hline & Total & 30 & 100 \\
\hline \multirow[t]{3}{*}{ UGI } & Grade 0 & 18 & 60 \\
\hline & Grade 1 and above & 12 & 40 \\
\hline & Total & 30 & 100 \\
\hline \multirow[t]{3}{*}{ LGI } & Grade 0 & 9 & 30 \\
\hline & Grade 1 and above & 21 & 70 \\
\hline & Total & 30 & 100 \\
\hline \multirow[t]{3}{*}{ Rectum } & Grade 0 & 18 & 60 \\
\hline & Grade 1 and above & 12 & 40 \\
\hline & Total & 30 & 100 \\
\hline
\end{tabular}

3 toxicity requiring parenteral support. Grade 1 diarrhea developed in all the patients with GI toxicity after 3 weeks i.e., after $30 \mathrm{~Gy}$ and in the six patients who had grade 2 and 3 diarrhea, the toxicity was seen after $40 \mathrm{~Gy}$ of radiation.

\section{Vomiting:}

The second most common GI toxicity observed was vomiting. Of those who had vomiting $50 \%$ had grade 1 , $33.33 \%$ had grade 2 requiring oral antiemetics and $16.67 \%$ had grade 3 vomiting requiring parenteral support. The grade 2 and 3 vomiting seen in the six patients was seen after 36 Gy of radiation. The remaining 6 developed grade 1 toxicity after 30 Gy of radiation. Vomiting was seen usually 2 days after administration of chemotherapy suggesting that cisplatin a highly emetogenic drug probably contributed to the development of vomiting.

Rectal toxicity

The most common rectal toxicity observed was tenesmus which was seen after $20 \mathrm{~Gy}$. 75\% had grade 1 toxicity in the form of rectal pain not requiring analgesics. 3 patients developed bleeding per rectum after 30 Gy of 
RT. Procto-sigmoidoscopy in these patients revealed telangiectasia in the rectal mucosa. None of the patients had grade 3 rectal toxicity.

4) Hematological toxicity (Table 3)

Overall 6 patients (20\%) had hematological toxicity of which $16.7 \%$ had grade 1 and $3.3 \%$ had grade 3 toxicity. 83.33\% had grade 1 neutropenia which developed in these patients in the fourth week of treatment, after completion of four cycles of chemotherapy. One patient developed pancytopenia after 3 cycles of chemotherapy i.e., grade 2 neutropenia, grade 3 anemia requiring blood transfusion and grade 1 thrombocytopenia and so further chemotherapy was stopped for that patient.

\subsection{Dosimetric Parameters}

The mean Dmax for the PTV was $105.06 \%$ (SD 1.03). The mean absolute Dmax dose to PTV was 48.23 Gy (SD 0.62). The PTV mean dose was 47.02 Gy. The minimum Dmax to the PTV was $102.6 \%$ and the maximum was $105 \%$.

The mean rectal dose was 46.55 Gy (SD 1.10). The mean total volume of rectum contoured was 56.9 cc (SD 14.47) and median 53.7 cc. The mean volume of rectum within the PTV of rectum was 48.23 cc (SD 0.62 ). The rectal maximum dose to 2 cc volume was $47.91 \mathrm{~Gy}$.

1) Bowel bag:

The RTOG recommends bowel contouring as a single bowel bag which includes both small and large intestine. The mean total volume of the bowel bag was 1420.47 cc (SD 588.45) and median volume was 1213.95 cc. The mean volume of bowel in the PTV was 192.56 cc (SD 74.75).

2) Small bowel

The mean dose to small bowel was 26.09 Gy (SD 7.95, median 25.89) and the average maximum dose to 2 cc volume was $47.94 \mathrm{~Gy}$. The mean total volume was $992.63 \mathrm{cc}$ (SD 507.33, median $835.55 \mathrm{cc}$ ) and the mean volume of small bowel in the PTV was 142.33 cc (SD 76.12, median 158.8). The range of small and large bowel volume was high compared with other OARs possibly due to variation in the bowel filling between patients and also due to greater mobility of these organs inside the abdomen.

3) Large bowel

The mean large bowel dose was $19.42 \mathrm{~Gy}$ (SD 8.21). The large bowel maximum dose to 2 cc volume was 47.43 Gy (SD 1.36). The mean total volume of large bowel contoured was 427.84 cc (SD 211.67, median 339.6 cc). The mean volume of large bowel in the PTV was 47.21 (SD 46.08).

The mean volume of each organ spared by the use of conformal RT using MLC is given below: Rectum 21.56 cc (SD 15.73 cc, median 16.7 cc), Small bowel 850 cc (SD 495.8, median 705.9 cc), bone marrow 633 cc (SD

Table 3. Status of hematological toxicity.

\begin{tabular}{cccc}
\hline Parameter & Status of toxicity & Frequency & Percentage \\
\hline Neutropenia & Grade 0 & 24 & 80 \\
& Grade 1 and above & 6 & 20 \\
Anemia & Total & 30 & 100 \\
& Grade 0 & 29 & 3.3 \\
& Grade 1 and above & 1 & 100 \\
Thrombocytopenia & Total & 30 & 93.3 \\
& Grade 0 & 28 & 6.7 \\
Hematologic toxicity (overall) & Grade 1 and above & 2 & 100 \\
& Total & 30 & 80 \\
\hline
\end{tabular}


85.26, median 626.9 cc), large bowel 377.9 cc (SD 194.6, median 324 cc) and bladder 126.7 cc (SD 106.8 cc, median $84.7 \mathrm{cc}$ ).

\section{Discussion}

The basic aim of conformal radiotherapy is to escalate the dose to the target volume while protecting the organs at risk (OARs) in order to reduce normal tissue toxicity. The ICRU 62 recommends that the tumor and the nodal PTV be covered between $95 \%$ to $107 \%$ of the prescribed dose. i.e., the dose inhomogeneity across the PTV is maintained between $-5 \%$ to $+7 \%$ [7]. Forrest et al. took $95 \%$ of the PTV to receive $100 \%$ of the dose as ideal. The Dmax was taken as $105 \%$ and if more than $99 \%$ of the PTV is covered by the $95 \%$ isodose line then the plan was accepted [4]. In our study we have tried to improve the PTV coverage (99\% of PTV covered by at least 99\% isodose) while maintaining the Dmax at 105\%. By using subfields and adjusting beam weight ages we brought down the PTV maximum dose (Dmax) to around 105\% while achieving increased target coverage. Achieving this goal was time consuming and labor intensive. We tried to analyze whether such tight optimization reflects in a reduction in acute toxicity and compliance of the patient to EBRT because in our center acute toxicity was one of the major reasons for dropouts from treatment during EBRT. Macdonald et al. assessed the RTOG acute radiation morbidity scoring criteria every week taking the single worst score in any category. A similar method of toxicity assessment was employed in or study [8].

In the Cochrane met-analysis published in 2005, acute toxicity was analyzed in detail in ten published trials [9]. Out of all the ten published trials, three trial designs (Keys, Rose and Pearcey et al.) were similar to our study in terms of chemotherapy prescribed to the patient while the dose and technique of radiation differed. We analyzed the results of our study in comparison to these three landmark randomized trials [10]-[12].

Acute GI toxicity was lesser in our study especially grade 2, 3, 4 when compared to other studies. In our study most of the patients had only grade 1 GI toxicity. The occurrence of GI toxicity in the other studies was as follows: Keys (grade $1-31.1 \%, 2-31.1 \%, 3-26.8 \%, 4-4.9 \%$ ), Pearcey (grade 3-8.07\%, 4-4\%) and Rose (grade 1-18.2\%, 2-15.9\%, 3-4.5\%, 4-2.3\%) [10]-[12]. Grade 2, 3 and 4 lower GI toxicity was much lesser in our study (43.3\% had grade 1, 20\% had grade 2 and $6.7 \%$ had grade 3 toxicity.

In our study we found that the most common toxicity was GI toxicity, diarrhea being the most common GI toxicity followed by vomiting.

Majority of our patients had Grade 1 skin reactions (36.7\%). There was no acute grade 3 and 4 skin toxicity in our study whereas Keys ( $3.8 \%$ grade $3,0 \%$ grade 4$)$, Pearcey (2.4\% grade $3,0 \%$ grade 4 ) and Rose $(0.6 \%$ grade $3,0.6 \%$ grade 4 ) showed some grade 3 skin toxicity. Rose study also reported a $0.6 \%$ grade 4 skin toxicity. The occurrence of grade 2 toxicity was also lesser in our study 6.7\% vs. 9.8\% (Keys) [10]-[12].

There was no grade 2, 3 and 4 genitourinary toxicity in our study whereas Keys (grade $1-23.5 \%$, 2-23.5\%, $3-7.7 \%$, 4-1.1\%), Pearcey (grade 3-2.4\%, 4-0\%) and Rose (grade $1-6.3 \%, 2-3.4 \%, 3-1.7 \%$, 4-1.1\%) showed higher toxicity for all the grades compared with our study. Only grade 1 toxicity was seen in our study. The occurrence of grade 1 toxicity was $3.33 \%$ in our study. A $44.5 \%$ reduction in the volume of bladder within the treated volume was achieved in our study while keeping Dmax around 105\% which reflected in the reduced genitourinary toxicity.

The overall hematological toxicity in our study was much less compared to Keys and Pearcey et al.. Only one patient in our study had grade 3 (3.33\%) hematological toxicity vs. $36.1 \%$ grade 3 and $3.2 \%$ grade 4 (Keys) and $4.8 \%$ grade 3 and $0 \%$ grade 4 (Pearcey). The grade 1 toxicity in our study was $16.66 \%$ vs. $19.7 \%$ Keys [10] [11]. Only one (4.54\%) patient developed local or regional recurrence and hence the dose optimization 3DCRT in our study probably resulted in better local control. On follow up one patient developed multiple bone metastasis and one patient developed lung and supraclavicular nodal metastasis.

In our study $25 \%$ of rectum, $8.4 \%$ of small bowel and $44 \%$ bladder could be spared from the PTV. Notably $84 \%$ of bone marrow could be spared from the PTV by the use of 3DCRT. However these measurements were made from the pre-treatment contoured images of the patients and everyday change in the volume of mobile organs like bowel loops and bladder within the PTV has to be taken into consideration.

In our study the contouring, planning and plan verification was done by the same oncologist and physicist and hence inter-observer bias was reduced. Setup verification was done for all the patients on the first three days of treatment and then weekly once ensuring minimal setup errors. Organ motion, target delineation inaccuracies due to poor imaging and longer planning time are some of the problems that we faced in our study. The lack of a comparative arm is another limitation of our study. The use of subfields necessitated more frequent setup verifi- 
cation. Verification of the subfields can be difficult and sometimes arbitrary. Portal images of the treatment fields may improve the accuracy of delivery of radiation using the subfields. The planning process was laborious and time consuming. The use of concurrent chemotherapy was a confounding factor in analyzing the toxicity and the increased toxicity seen in a few patients could have been due to chemotherapy.

However dose optimization resulted in reduced toxicity which reflected in better compliance and there were no treatment dropouts in our study.

\section{Conclusion}

3DCRT with dose optimization is feasible in cervical cancer and results in a better toxicity profile than conventional radiotherapy. In comparison with historical data our study demonstrated a decrease in grade 2, 3 and 4 acute toxicities. The advantage of reduced toxicity reflected clearly in the patient compliance to treatment and there were no dropouts in our study due to increased toxicity.

\section{References}

[1] Parkin, D.M., Bray, F., Ferlay, J. and Pisani, P. (2001) Estimating the World Cancer Burden: Globocan 2000. International Journal of Cancer, 94, 153-156.

[2] Green, J., Kirwan, J., Tierney, J., Vale, C., Symonds, P., Fresco, L., et al. (2005) Concomitant Chemotherapy and Radiation Therapy for Cancer of the Uterine Cervix. Cochrane Database of Systematic Reviews, 3, Article ID: CD002225. http://dx.doi.org/10.1002/14651858.cd002225.pub2

[3] Chan, P., Yeo, I., Perkins, G., Fyles, A. and Milosevic, M. (2006) Dosimetric Comparison of Intensity-Modulated, Conformal, and Four-Field Pelvic Radiotherapy Boost Plans for Gynecologic Cancer: A Retrospective Planning Study. Radiation Oncology, 1, 1-13. http://dx.doi.org/10.1186/1748-717X-1-1

[4] Forrest, J., Presutti, J., Davidson, M., Hamilton, P., Kiss, A. and Thomas, G. (2012) A Dosimetric Planning Study Comparing Intensity-Modulated Radiotherapy with Four-Field Conformal Pelvic Radiotherapy for the Definitive Treatment of Cervical Carcinoma. Clinical Oncology, 24, 63-70. http://dx.doi.org/10.1016/j.clon.2011.06.008

[5] Gerstner, N., Wachter, S., Knocke, T.H., Fellner, C., Wambersie, A. and Pötter, R. (1999). The Benefit of Beam’s Eye view Based 3D Treatment Planning for Cervical Cancer. Radiotherapy \& Oncology, 51, 71-78. http://dx.doi.org/10.1016/s0167-8140(99)00038-9

[6] Radiation Therapy Oncology Group (RTOG). https://www.rtog.org/CoreLab/ContouringAtlases/GYN.aspx

[7] ICRU-62 (1999) ICRU Report 62 International Commission on Radiation Units and Measurements. Bethesda, MD.

[8] Macdonald, D.M., Lin, L.L., Biehl, K., Mutic, S., Nantz, R. and Grigsby, P.W. (2008) Combined Intensity-Modulated Radiation Therapy and Brachytherapy in the Treatment of Cervical Cancer. International Journal of Radiation Oncology*Biology*Physics, 71, 618-624. http://dx.doi.org/10.1016/j.jjrobp.2008.02.014

[9] Green, J., Kirwan, J., Tierney, J., Vale, C., Symonds, P., Fresco, L., et al. (2005) Concomitant Chemotherapy and Radiation Therapy for Cancer of the Uterine Cervix. Cochrane database of Systematic Reviews, 3, Article ID: CD002225. http://dx.doi.org/10.1002/14651858.cd002225.pub2

[10] Keys, H.M., Bundy, B.N. and Stehman, F.B. (1999) Cisplatin, Radiation, and Adjuvant Hysterectomy Compared with Radiation and Adjuvant Hysterectomy for Bulky Stage IB Cervical Carcinoma. The New England Journal of Medicine, 340, 1154-1161. http://dx.doi.org/10.1056/NEJM199904153401503

[11] Pearcey, R., Brundage, M., Drouin, P., Jeffrey, J., Johnston, D., Lukka, H., et al. (2002) Phase III Trial Comparing Radical Radiotherapy with and without Cisplatin Chemotherapy in Patients with Advanced Squamous Cell Cancer of the Cervix. Journal of Clinical Oncology, 20, 966-972. http://dx.doi.org/10.1200/JCO.20.4.966

[12] Rose, P.G., Bundy, B.N., Watkins, E.B., Thigpen, J.T., Deppe, G., Maiman, M.A., et al. (1999) Concurrent Cisplatin-Based Radiotherapy and Chemotherapy for Locally Advanced Cervical Cancer. The New England Journal of Medicine, 340, 1144-1153. http://dx.doi.org/10.1056/NEJM199904153401502 